\title{
Making sense of binary metal alloys
}

A Cray-led attack on the structure of the binary alloy of nickel and platinum seems to be most easily interpreted by reference to empirical rules put forward by Hume-Rothery more than half a century ago.

WILliam Hume-Rothery, the metallurgist who in the 1930s did much to develop rules for the structure of metallic alloys based on what was then being learned of electronic structure, had the misfortune to be stone-deaf. Among other things, his affliction meant that, over several decades, he was not invited to be a member of an Oxford Senior Common Room until the late A. S. Russell, once active in early radioactivity (with Fajans and Soddy, for example) persuaded the members of the college called Christ Church — it is also a cathedral - that they could stomach the occasional social embarrassment of a deaf man at dinner.

By then, 1944 or thereabouts, HumeRothery had become skilled at using his rasping monotone to get his points across; many cohorts of students were persuaded that what matters in the determination of the structure of an alloy, its electrical conductivity and even its inclination towards order or disorder, are differences of atomic (not ionic) size, electronegativity and the electron-per-atom ratio. One of his rules, for example, is that atoms differing in size by more than 15 per cent will not form solid solutions, which rather begs the question of what is meant by "size" when atoms may be embedded as ions in a sea of moveable electrons. Nevertheless, Hume-Rothery claimed no more for the rules than that they were empirical generalizations cast in a language ripe for explanation. Yet the explanations have been slow to come.

There is, for example, the question of when an equimolar binary alloy is likely to form crystals in which neighbouring lattice sites are alternately occupied by different species of atoms. The question is obviously of practical importance, given that a material such as ordered $\beta$-brass is evidently very different from its components, copper and zinc. Conventional wisdom (acquired in the past decade, after HumeRothery's time) is that ordering in transition-metal alloys is probable when the $d$ conduction band is roughly half filled by electrons, and is improbable when the Fermi energy (that of the most energetic electron) is near either the top or the bottom.

Now F. J. Pinski and five other theoreticians have dealt in some detail with what appears to be an exception to that rule the equimolar alloy of nickel and platinum, $\mathrm{Ni}_{0,5} \mathrm{Pt}_{i 5,5}$, which has a $d$-band system almost filled with electrons, but whose structure has a high degree of shortrange order. Part of the interest of their article is that it attempts, apparently successfully, to calculate the influence of Hume-Rothery's three factors.

For what it is worth, alloys of $\mathrm{Ni}$ and $\mathrm{Pt}$ are ferromagnetic at sufficiently low concentration of $\mathrm{Pt}$, which has made the system a test-bed for studies of the interaction between chemical and magnetic ordering. (These days, no doubt, the possibility that a similar interplay is involved in the influence of doping cations in hightemperature superconductors should not be overlooked.) But the paper of Pinski et al. (Phys. Rev. Lett. 66, 766; 1991) is also remarkable in that its six authors give six different affiliations (the universities of Cincinnati, Messina, Warwick and Bristol, the Sandia National Laboratory at Livermore and the Oak Ridge National Laboratory in Tennessee).

Pinski et al. choose to tackle the problem from the difficult end of the spectrum, by treating first the case of a randomly disordered solid solution of $\mathrm{Ni}$ and $\mathrm{Pt}$, and estimating its instability with respect to microscopic transformations that lead to short-range or local order. But even if they had set out in the other direction to estimate the instability of the ordered phase with respect to disordering processes (entailing assumptions, perhaps invalid, about the structure of the ordered phase), they would have had to devise some way of representing self-consistently the electrical potential to which the $d$ electrons are subjected on account of the chance that a lattice-site occupied by $\mathrm{Ni}$ in the ordered state may, in reality, be occupied by Pt.

The trick, called the coherent potential approximation, is a kind of mean-field theory which, like all mean-field theories, is characterized by the level in the argument at which the averaging is carried out. Plainly it would make no sense to suppose that the electrical potential emanating from each lattice site is the average of the potentials of the cores of $\mathrm{Ni}$ and $\mathrm{Pt}$, for that would exclude the possibility of short-range ordering. Moreover, because the likelihood of short-range ordering is critically a function of the temperature, it is as well to include the lattice vibrations from the start. The result is a way of handling the electronic potential both as a determinant of the degree of short-range order and as a consequence thereof.
Interestingly, while thanking both the Ohio Supercomputer Center and Cray Research Inc., Pinski et al. present their results as a two-dimensional plot of the short-range order parameter for various directions in reciprocal-lattice space and find themselves following HumeRothery in the manner in which they say what it means. But one conclusion is that the predominant effect in the ordering process is that of nearest neighbours (and that the energetic consequences of nextnearest neighbours are only 10 per cent as great).

Another striking feature of the calculation is that $\mathrm{Pt}$ atoms contribute to the random alloy electron states chiefly in the lower half of the $d$-electron band, with $\mathrm{Ni}$ atoms supplying the remainder. The argument then goes that, in the real world, states from the two sources will hybridize with each other, forming energetically favourable chemically bonding states. This, Pinski and his colleagues say, is the source of the ordering propensity in NiPt. And as the states that hybridize, or mix, in the ordered structure are mostly both below the Fermi energy (which measures the extent to which the band is filled), the exact location of the Fermi level is not particularly relevant to the ordering propensity. That, the authors say, explains why the half-full band criterion - last decade's received wisdom - is irrelevant, in this exceptional case, to the ordering propensity of NiPt. In Hume-Rothery language, this is a way of saying that the electron/atom ratio is immaterial.

Pinski et al. go one step further towards Hume-Rothery's position in taking up the question of the difference of size between the $\mathrm{Ni}$ and $\mathrm{Pt}$ atoms in the ordered alloy. In the quantum picture of a lattice structure held together by electrons, the 'size' of the entities occupying neighbouring lattice sites has no meaning on its own. What the calculation (and the real world) provide is the lattice spacing, which is a function of the crystal structure as a whole, in which the degree of shortrange ordering is merely another parameter. But in the randomly ordered NiPt alloy, $\mathrm{Ni}$ atoms occupy more space, and the larger Pt atoms less space, than in the respective pure metals. So short-range ordering, where one kind of atom tends to be surrounded by atoms of the other kind, here tends to relieve strain. Thus in principle, if not in detail, is Hume-Rothery vindicated again.

John Maddox 